\title{
La Trascendencia del Banco Mundial en La Ley General del Servicio Profesional Docente. Elementos para el Análisis
}

DOI: https://doi.org/10.32870/dse.v0i9.295

\section{Lucía Mantilla Gutiérrez*}

\begin{abstract}
Resumen: Este texto analiza la trascendencia del Banco Mundial y del Grupo del Banco Mundial en el campo educativo desde la convención mundial que en el año 1999 logró el acuerdo llamado Educación Para todos. En particular se examina la propuesta del Grupo del Banco Mundial denominada Systems Approach for Better Education Results (SABER, por sus siglas en inglés) y más específicamente Saber-Teachers que contiene 8 metas, todas ellas presentes en la Ley General del Servicio Profesional Docente promulgada en México el 11 de septiembre de 2013. Concluye el trabajo con algunas de las críticas académicas a las políticas educativas del Banco Mundial. Palabras claves. Banco Mundial. Reformas globales y gerenciales en el campo educativo. Ley General del Servicio Profesional Docente.
\end{abstract}

\begin{abstract}
In this paper I analyze the transcendence the World Bank and the World Bank Group have had in the education field since the world convention held in Jomtien, Thailand in 1990, whose agreement took the name of Education For All. I particularly analyze the eight goals of SABER Teachers which are all present in the General Law of Professional Teaching Service of Mexico enacted on September 11th, 2013. I conclude by outlying some of the academic criticism that has been made toward the educational policies of the World Bank.
\end{abstract}

\section{Introducción}

La palabra trascendencia, contenida en el título de este trabajo, resulta conveniente para destacar que la influencia del Banco Mundial y más recientemente del Grupo del Banco Mundial no se agota en aspectos económicos o financieros, sino que abarca la dimensión de lo político; es decir, de lo discursivo e intangible que concierne a la atribución del sentido de la educación misma; y se materializa también en la política educativa, en programas educativos que afectan la totalidad de los aspectos vinculados con la educación e incluso, como ha sido estudiado en otros países, las prácticas cotidianas en el aula, la subjetividad de los docentes y el clima escolar.

En términos generales, el Banco Mundial es un organismo conformado por 188 países accionistas cuyo número de votos es determinado por la riqueza de cada uno de ellos. El Banco Mundial está compuesto por el Banco Internacional de Reconstrucción y Fomento (BIRF) que brinda asesoría y préstamos a países de mediano ingreso y capacidad crediticia, entre los cuales se encuentra México y por la Asociación Internacional de Fomento (AIF) que otorga préstamos de bajo interés y con largos periodos de amortización. En Latinoamérica solamente 9 países y pequeñas economías

* Profesora investigadora del Departamento de Estudios en Educación de la Universidad de Guadalajara. Correo electrónico: luciamantill@gmail.com 
insulares están en la lista de países elegibles para este último tipo de préstamos. En la página web del Banco Mundial se informa que "en la actualidad, 82 países pueden tener acceso a recursos de la AIF, los cuales en conjunto albergan a 2.800 millones de personas, la mitad de la población total del mundo en desarrollo. De esa cifra, se estima que 1.800 millones viven con ingresos de US\$2 al día o menos".

Por otra parte, el Grupo del Banco Mundial contiene al Banco Mundial e incluye, además, las siguiente tres instituciones: el Organismo Multilateral de Garantía de Inversiones; el Centro Internacional de Arreglo de Diferencias Relativas a Inversiones y finalmente, la Corporación Financiera Internacional que otorga préstamos al sector privado.

En este trabajo se reflexiona sobre la creciente importancia del Banco Mundial (en adelante BM) y del Grupo del Banco Mundial (en adelante GBM) en las dos más importantes convenciones mundiales sobre educación, la que tuvo lugar en Jomtien, Tailandia y cuyo acuerdo tomó el nombre Educación para Todos (1990). Esta misma declaración básicamente se renueva en términos conceptuales en el Foro Mundial de la Educación que se llevó a cabo en Dakar, la capital de Senegal, en el año 2000, mismo año en el que se establecieron las metas del mileno.

Ahora bien, la estrategia para el 2020 denominada Learning For All o Aprendizaje Para Todos que comienza circular en el año 2011, es autoría del Grupo del Banco Mundial.

El apoyo financiero de ambos organismos para la educación ha aumentado desde que se establecieron las metas del milenio y desde el año 2001 cuando la Corporación Financiera Internacional comenzó a centrarse en el sector educativo, ha invertido \$500 millones de dólares en 46 proyectos de educación privada (Learning For All, 2011: 1).

Otra propuesta que se debatirá en el año 2015 es la de la Organización de las Naciones Unidas para la Educación, Ciencia y la Cultura (UNESCO) titulada Educación Ante Todo (2012), aunque no es incluida en el análisis de este trabajo conviene brevemente mencionar que en ella se concibe a la educación como un derecho fundamental y un proceso de formación humana integral en tres ámbitos prioritarios: 1-. Escolarizar a todos los niños. 2.- Mejorar la calidad del aprendizaje. 3.Fomentar la conciencia de ser ciudadanos del mundo.

Sin duda, tanto la propuesta del GBM como la de la UNESCO constituyen perspectivas que se debatirán y afectarán, a nivel global, los acuerdos que se tomen en el próximo 2015. Después de todo, la trascendencia de la que hablamos no se limita a México. Como subrayan Antoni Verger y Hülya Kosar Altinyelken tanto como los autores que ellos citan (2013) estas llamadas Global Managerial Education Reforms en las que el BM y el GBM son los principales promotores no viajan en "paquete", más bien se aplican de modo selectivo; esto es, arriban a contextos específicos donde

\footnotetext{
${ }^{1}$ Los países son: Bolivia, Dominica, San Vicente, Santa Lucia, Granada, Guayana, Haití, Honduras y Nicaragua; existen diferencias entre ellos, que no conciernen al objeto de esta presentación. Véase Países prestatarios de la AIF en http://www.worldbank. org/ida/borrowing-countries-spanish.html. Consultado el 5 de noviembre de 2013.
} 
son "re contextualizadas" ya que en su aplicación se vincula con la ideología política del gobierno en turno, el apoyo que logran alcanzar en la sociedad, las respuestas sindicales, etcétera.

Reformas y leyes similares a la mexicana han aterrizado, por decirlo así, en contextos histórica, social y culturalmente diferentes, es por tanto posible, en cierta forma, adelantar las consecuencias de la nueva Ley. Más específicamente, en Inglaterra, en Indonesia, en Jamaica, en Namibia, Turquía, en Chile, en Colombia, etcétera, no habitan ni la señora Gordillo, ni el SENTE ni la CENTE y aunque la preocupación por la educación suele tomar un tinte nacionalista, en muchas ocasiones atizado por las comparaciones internacionales, esto no alcanza para otorgar ni a su origen, ni a su contenido un arraigo nacional.

En orden de continuidad veremos que en la estrategia del GBM para el año 2020, llamada, Learning for All, se destaca una definición sistémica y multisectorial del sistema educativo y se anuncia que se están desarrollando instrumentos apropiados para dicho sistema, todos ellos bajo el nombre de Systems Approach for Better Education Results (SABER, por sus siglas en inglés) que constituye básicamente un marco del diagnostico y comparación de los sistemas educativos y se agrega que SABER contendrá herramientas para evaluar a estudiantes, al desarrollo infantil temprano, entre otros.

En este mismo documento, se plantea que SABER está en una fase inicial (Learning For All, 2011: 62) y que será realizado en cinco etapas: en la primera se desarrollará un marco conceptual que incluye metas e instrumentos; en la segunda etapa, se desarrollaran las herramientas de evaluación correspondiente y se construirán prototipos de esas herramientas; la tercera etapa consiste en comprometerse con las agencias gubernamentales y las partes interesadas de países seleccionados, para validar el marco conceptual y las herramientas marco y la evaluación; en la cuarta etapa se difundirán los resultados de las aplicaciones piloto y en la quinta se promoverá su aplicación global.

Ahora bien, SABER-Teachers ya ha sido lanzado como prototipo y se han realizado estudios piloto en algunos países seleccionados, para validar el marco teórico y las herramientas de evaluación (Learning For All, 2011: 62). Más precisamente, en la versión actualizada de abril 2013 SABER- Teachers - que como he señalado es una parte integral del Systems Approach for Better Education Results pero específicamente referida a los maestros - se menciona que se ha recopilado información o está recopilando en 65 sistemas educativos de 44 países, entre ellos México.

Para diciembre de 2013 SABER Teachers cuenta con una página WEB (http://saber.worldbank.org/index.cfm) en la cual puede verse el reporte de 20 países entre los cuales no se encuentra México; no obstante, SABER Teachers contiene 8 metas que, como veremos, se encuentran integradas en la reciente Ley.

Finalmente, me permitiré elaborar una síntesis de las críticas que la estrategia del BM ha generado en la comunidad académica mundial. 


\section{Antecedentes}

El uso de la palabra antecedentes, la incrustada temporalidad a la que remite aquí resulta problemática por varias razones. Claramente la trascendencia del BM comienza en el pasado, que aquí situaremos a partir de 1990, pero no solamente sucede antes, ni solamente se prolonga hasta el presente, sino que también anticipa el futuro de la próxima convención mundial sobre la educación, en la medida en la que las diversas perspectivas ya han comenzado a circular y debatirse, y quizás sobre todo, porque la nueva estrategia implica más bien una continuidad fortalecida conceptual y operativamente.

Así, el GBM se distancia de la definición tradicional de sistema educativo y subraya la perspectiva multisectorial y el enfoque más inclusivo que otorga al concepto, aunque simultáneamente, señala que en términos financieros, no contiene ninguna novedad. Más específicamente, a nivel nacional, el GBM se centrará en contribuir a las reformas de los sistemas educativos, pero el concepto sistema educativo incluye aquí todo el espectro de oportunidades de aprendizaje disponibles en un país, público y privado; los programas formales y no formales; todos los actores sociales involucrados; los docentes, los estudiantes y sus familias, etcétera. Incluye también las normas, las políticas y los recursos, los mecanismos de financiamiento y de rendición de cuentas (Grupo del Banco Mundial Aprendizaje Para Todos. Resumen Ejecutivo. 2011: 5).

El enfoque multisectorial implica que "Los resultados de la educación también están influenciados por las políticas que no están directamente bajo la competencia de los ministerios o agencias de educación; por ello el apoyo hacia la educación debe contemplar programas que pertenecen al sector salud, protección social, empleo, transporte, agua, sector público gobernanza, entre otros. Pero en el mismo documento se señala que "Esto no es un nuevo modelo de negocio. En el año fiscal 2009, más del 40 por ciento de nuevos préstamos para la educación formaba parte de otro sector de operación" (Learning For All, 2011: 67).

Si bien el uso del término antecedentes es problemático, lo cierto es que es posible situar la creciente importancia del BM en asuntos educativos a partir de la conferencia mundial en Tailandia 1990, que fue auspiciada por la Organización de las Naciones Unidas para la Educación, la Ciencia y la Cultura (UNESCO), el Fondo de las Naciones Unidas para la Infancia (UNICEF); el Programa de Naciones Unidas para el Desarrollo (PNUD) y el propio BM. Allí 155 gobiernos suscribieron la declaración y el marco de acción llamado Educación para Todos que, como demuestra Torres (2000: 6) al analizar detalladamente su contenido no sólo pretendía asegurar educación básica de calidad para niños, jóvenes y adultos, sino también renovar la visión y el alcance de la educación.

La misma autora señala que el PNUD no defendió una posición definida en tanto que la UNESCO abogó por una comprensión amplia de lo educativo y por la inclusión de la alfabetización y la educación de adultos en particular; el UNICEF defendió el desarrollo infantil y la educación inicial dentro del concepto de educación básica, así como - junto con la UNESCO- la necesidad de flexibilizar y diversificar la oferta educativa incluyendo variantes no-formales; es decir, distintas de 
las variantes escolares convencionales; el BM, en cambio, propuso focalizarse en el sistema escolar y en la educación primaria.

La misma autora señala que el ideario y las metas de Educación Para Todos a las que llama la visión ampliada, en tanto que no se centraba en la escolarización y era novedosa y potencialmente transformadora, se encogió en la medida que, en términos reales, se focalizó solamente en la educación primaria y eliminó la diversidad que pretendía, restringiéndose al cumplimiento de 18 indicadores cuantitativos. Así, lejos de los propósitos, las políticas globales fueron sumamente similares en todo el mundo, reconocibles tanto en Asia como en África, América Latina y el Caribe, de modo que

La uniformación de la política educativa a escala global está vinculada al creciente peso de los organismos internacionales en el diseño y ejecución de la política educativa en los países en desarrollo, particularmente del Banco Mundial, el socio más fuerte de la Educación Para Todos y el que lideró el escenario educativo en la década de los 90 (Torres, 2000: 74).

Esta autora cita el análisis realizado por Coraggio y ella misma en 1997, que aquí sintetizo ya que nos permite ver con claridad la propuesta o visión ampliada y la manera en que, finalmente y en la práctica se restringió:

\begin{tabular}{|l|l|}
\hline \multicolumn{2}{|c|}{ Educación para Todos } \\
\hline \multicolumn{2}{|c|}{ Visión Ampliada } \\
$\begin{array}{l}\text { Educación para todos, niños, jóvenes y adultos; se } \\
\text { inicia en el nacimiento y dura toda la vida. }\end{array}$ & $\begin{array}{l}\text { Educación para los niños y niñas (los más pobres } \\
\text { entre los pobres). }\end{array}$ \\
\hline Educación básica, dentro y fuera del aparato escolar. & Educación escolar (primaria). \\
\hline $\begin{array}{l}\text { Necesidades básicas de aprendizaje diversas ya } \\
\text { que son diferentes en diversos grupos y culturas, } \\
\text { así como lo son también los diversos medios y } \\
\text { modalidades para satisfacerlas. }\end{array}$ & $\begin{array}{l}\text { Necesidades mínimas de aprendizaje, uniforme e igual } \\
\text { para todos. }\end{array}$ \\
\hline Concentrar la atención en el aprendizaje. & Mejorar y evaluar el rendimiento escolar. \\
\hline Ampliar la visión de la educación básica. & $\begin{array}{l}\text { Ampliar el tiempo (número de años) de la escolaridad } \\
\text { obligatoria. }\end{array}$ \\
\hline $\begin{array}{l}\text { Educación básica como cimiento de aprendizajes } \\
\text { posteriores. }\end{array}$ & Educación básica como un fin en sí misma. \\
\hline Mejorar las condiciones de aprendizaje. & $\begin{array}{l}\text { Mejorar las condiciones internas de la institución } \\
\text { escolar. }\end{array}$ \\
\hline Todos los países. & Los países en desarrollo. \\
\hline Responsabilidad de los países. & $\begin{array}{l}\text { Responsabilidad de los países (organismos } \\
\text { gubernamentales y no-gubernamentales) y la } \\
\text { comunidad internacional. }\end{array}$ \\
\hline
\end{tabular}

Fuente: Coraggio y Torres, La educación según el Banco Mundial. Un análisis de sus propuestas y métodos, Buenos Aires: CEMMiño y Dávila Editores, 1997. 
Ahora, bien diez años después, en el año 2000, se llevó a cabo en Dakar el Foro Mundial Educación Para Todos, con el propósito de evaluar los alcances de la convención mundial de 1990 y establecer un nuevo marco de acción, Rosa María Torres fue panelista en este foro Mundial y puede leerse su propia experiencia en el texto ¿Qué pasó en el Foro Mundial de la Educación? (2000) en donde refiere la cerrada forma de participación, el desánimo prevaleciente y los alcances logrados a nivel mundial que pueden verse en la siguiente tabla:

\begin{tabular}{|l|l|l|}
\hline \multicolumn{1}{|c|}{ 1990-2000: Algunos Datos Comparativos } & \multicolumn{1}{c|}{$\begin{array}{c}\text { 1990 } \\
\text { (Jomtien) }\end{array}$} & \multicolumn{1}{c|}{$\begin{array}{c}\text { 2000 } \\
\text { (Dakar) }\end{array}$} \\
\hline $\begin{array}{l}\text { Gasto por alumno como porcentaje del Producto } \\
\text { Nacional Bruto (PNB) per cápita. }\end{array}$ & entre 6\% y 19\% & entre 8\% y 20\% (1998). \\
\hline $\begin{array}{l}\text { Niños y niñas en programas de educación inicial } \\
\text { ( a 6 años). }\end{array}$ & 99 millones & $\begin{array}{l}104 \text { millones (de un total de más } \\
\text { de } 800 \text { millones). }\end{array}$ \\
\hline Niños y niñas en la escuela. & 599 millones & $\begin{array}{l}681 \text { millones (44 millones de ese } \\
\text { aumento corresponde a niñas). }\end{array}$ \\
\hline Niños y niñas al margen de la escuela. & 106 millones & 117 millones (60\% niñas). \\
\hline Adultos analfabetos. & 895 millones & 880 millones (60\% mujeres). \\
\hline Tasa de alfabetización. & 0.75 & $\begin{array}{l}80 \% \text { (85\% hombres, } 74 \% \\
\text { mujeres). }\end{array}$ \\
\hline
\end{tabular}

La misma autora señala, a manera de síntesis, que entre Jomtien y Dakar hubo no sólo incumplimiento de las metas sino incluso estancamiento y empobrecimiento del discurso y del ideario original de Educación Para Todos; así, la "visión ampliada" de la educación básica esbozada en Jomtien permanece, de hecho, como un desafío vigente y pendiente, a desarrollar conceptualmente y a plasmar operativamente en los años venideros.

En la síntesis de los avances logrados en el marco de Educación para Todos, documento elaborado por Malcolm Skilbeck (2000) para la UNESCO, se destacan dos momentos respecto a México: el primero de ellos bajo el subtitulo Planes estratégicos y Medidas Legales refiere al Acuerdo Nacional para la Modernización de la Educación Básica en 1992, las reformas constitucionales y la nueva ley de educación de 1993, durante el gobierno de Carlos Salinas de Gortari. Se destaca que la reforma más importante fue la descentralización de la educación básica y la formación docente. Así, los objetivos generales, la definición de evaluación de contenidos y las políticas compensatorias fueron retenidos por el gobierno central, mientras que la administración del funcionamiento de las instituciones de educación pasó a manos de los Estados (Skilbeck, 2000: 21); por otra parte, México ocupó el primer lugar en la tasa bruta de matrícula en la educación preescolar o de la primera infancia; se menciona que casi $80 \%$ de los niños entre 3 y 5 años asisten al preescolar por un año o más (Skilbeck, 2000: 24). 
En la misma página de la Unesco ${ }^{2}$ se presentan los informes entregados por cada país y se puede acceder al documento titulado "México Evaluación de Educación para Todos, 2000" elaborado por la Dirección General de Relaciones Internacionales de la Secretaría de Educación Pública en enero del 2000, que contiene una sección descriptiva en la cual se destaca la reforma del artículo 3 constitucional que definió las obligaciones de la federación y de los Estados, que también incorporó la educación secundaria a la educación básica y estableció el imperativo de la integración de las personas con necesidades educativas especiales en los ámbitos educativos regulares. Destaca también que se han llevado a cabo acciones vinculadas con la equidad de género, la educación intercultural bilingüe y se ha mejorado el Programa de Educación a Distancia, como también el que por primera vez se ha instalado un Consejo Nacional de Participación Social en la Educación.

Más importante para el objeto de este trabajo es que este documento muestra como avance de las metas propuestas en Educación Para todos de 1990, lo que, entre otros, constituye el antecedente de la Ley General del Servicio Profesional Docente promulgada en septiembre del 2013: la instauración de la Carrera Magisterial, la creación de un sistema de monitoreo de la calidad educativa mediante la consolidación del Sistema Nacional de Evaluación.

Quisiera señalar que si bien el Foro Mundial de Dakar reafirma las metas planteadas en Educación para Todos aprobadas en Tailandia diez años antes, y se hace evidente el cambio económico en su política, ya que a partir de allí se le ha inyectado al acuerdo Educación para todos presupuestos sin precedentes a nivel mundial. También, como vimos, el GBM incrementó a partir de allí sus préstamos educativos al sector privado.

Pero quisiera destacar en particular, que en el año 2002 se creó una iniciativa de vía rápida inscrita en el marco de los acuerdos de Educación para Todos y vinculada con las Metas del Milenio: la Alianza Mundial para la Educación o Global Partnership for Education, (GPE) que se compone de cerca de 60 países en desarrollo, gobiernos donantes, organizaciones internacionales, el sector privado, profesores y grupos de la sociedad civil y ONG. GPE ha destinado US\$ 3,7 billones de dólares americanos en subsidios a la educación entre los años 2003 y 2013, desde 2003 los socios GPE han ayudado a que casi 22 millones más de niños vayan a la escuela, incluyendo 10 millones de niñas, ha contribuido para entrenar a unos 300.000 profesores, a construir, rehabilitar y equipar cerca de 53.000 aulas, comprado y distribuido unos 50 millones de libros de texto³.

Debe agregarse que los países más pobres (entre los cuales no se encuentra México) beneficiados con estos apoyos típicamente cubren entre $70 \%$ y $80 \%$ de los costos de los programas de educación (Véase Global Partnership for education. The Case for Investment, 2011-2014: 9).

Roseli y Steer (2013) dan cuenta del informe de seguimiento de Educación para Todos (UNESCO, 2012 b) que el gasto interno fue, por mucho, la más importante fuente de financiamiento contabilizada en $\$ 25$ billones; los restantes $\$ 3$ billones vinieron de los recursos provenientes de

\footnotetext{
${ }^{2}$ Véase http://www.unesco.org/education/wef/countryreports/mexico/contents.html.

${ }^{3}$ Véase http://www.globalpartnership.org/10-key-data-results.
} 
donantes. A pesar de ello, la ayuda internacional ha desempeñado un papel particularmente importante en países donde los recursos nacionales son demasiado escasos. Se estima que costará un total de $\$ 54$ billones anuales proporcionar educación básica para todos en 2015, en 46 países de bajos ingresos y países de ingresos medio-bajo. Suma superior a la del 2010, cuando se gastó un total de \$28 billones en educación básica; es por ello que el debate en la próxima reunión del 2015 donde se establecerán los compromisos internacionales y el destino de la educación es actualmente muy importante.

En el marco de estos datos, es importante subrayar que si bien la gran mayoría de los países de América Latina no son elegibles para donaciones internacionales, estas convenciones internacionales sí han afectado la política educativa, como veremos más adelante, donde se podrá observar la equivalencia entre La Ley General del Servicio Profesional Docente (2013) y SABER- Teachers del Banco Mundial.

Ahora bien, en SABER-Teachers (en el documento citado a lo largo de este trabajo: Working Paper Series. Número 4 de abril 2013 del Banco Mundial: 10) se afirma que este documento es fruto del equipo que ha consultado con expertos de diversas organizaciones, funcionarios gubernamentales y otros colegas del BM durante un periodo de tres años, que comenzó en febrero 2009.

Debe considerarse que, en términos generales, las mismas metas del BM en SABER Teachers se encuentran en el Acuerdo de Cooperación México-OCDE, de septiembre 2010, para mejorar la calidad de la educación de las escuelas mexicanas, titulado Mejorar las escuelas: estrategias para la acción en México. Así, las recomendaciones de la OECD Hacia una reforma educativa en México (2010: 6) son:

1. Definir la enseñanza eficaz. 2. Atraer a los mejores aspirantes. 3. Fortalecer la formación inicial docente.

4. Mejorar la selección docente. 5. Abrir todas las plazas a concurso. 6. Crear períodos de inducción y de prueba. 7. Mejorar el desarrollo profesional. 8. Evaluar para ayudar a mejorar. 10. Profesionalizar la formación y la asignación de plazas a los directores. 11. Fortalecer el liderazgo instruccional [sic] en las escuelas. 12. Aumentar la autonomía escolar. 13. Garantizar el financiamiento para todas las escuelas. 14. Fortalecer la participación social. 15. Crear un Comité de Trabajo para la Implementación.

Puede decirse que los expertos de ambas instituciones comparten una misma perspectiva teórica que, en sentido estricto, no puede considerarse "neoliberal", ya que le regresan el mando de la educación al Estado; son más bien reformas gerenciales, financieras, empresariales, de contraloría y auditoría (véase Verger et al., 2010); no obstante, en este trabajo no centro la atención en la OECD porque México ingresa a la OECD en 1994 y porque la trascendencia del BM en el campo educativo, no se limita a los países miembros de la OECD.

Antes de comenzar con la reflexión sobre la Ley recientemente aprobada, quisiera agregar que resulta sorprendente que el tema de la incidencia del Banco Mundial en el campo educativo mexicano y en particular en relación con los profesores resulte aún polémica, puede verse una 
manifestación de esta polémica en el texto titulado ¿Protestar por ser evaluados? escrito por David Calderón, director general de Mexicanos Primero, una organización que bajo el patronato de Emilio Azcárraga y otros ciudadanos mexicanos, algunos de ellos en la lista de millonarios de Forbes ${ }^{4}$, se define a sí misma como una iniciativa ciudadana independiente y plural. El autor escribe en el blog de dicha organización en enero de 2012, pero puede verse allí todavía, después de la promulgación de la Ley General del Servicio Profesional Docente. Calderón menciona que entre los argumentos que esgrimen los maestros en contra de la evaluación es que ésta es una imposición del Banco Mundial y responde a este argumento en el tono neutral de la tercera persona "da ternura por ingenuo" y agrega "El Banco Mundial, el FMI, el BID o cualquier otra agencia internacional difícilmente "impone" [sic] ya que si bien hacen recomendaciones, cientos de volúmenes de estas se quedan en los estantes de las oficinas. Al agregar textualmente la cita del texto se puede comprender el tono desde el que fue escrito

Es triste que algunos maestros de educación básica piensen que en México no hay especialistas de educación, universitarios renombrados internacionalmente, que propongan el valor de la evaluación docente, y que se imaginen "mandatos" de torvos multimillonarios quienes, desde sus oficinas de Washington, llamaron con sus teléfonos de oro a sus lacayos mexicanos para que a la de ya, les apliquen una evaluación a los maestros oaxaqueños que están poniendo en peligro sus bonos de NASDAQ. Ajá.

Éstas, en mi opinión, inmoderadas imágenes y expresiones parecen escritas desde alguna isla peculiar, desde donde primero, se pone en duda la legitimidad misma del Banco Mundial, en tanto que esta agencia argumenta que sus políticas derivan de una amplia consulta que incluye justamente a los organismos de la sociedad civil de la que el autor es director; segundo, cuestiona la importancia que el propio BM se atribuye a sí mismo como asesor en asuntos educativos a nivel global y la obligatoriedad que los préstamos educativos conllevan y que, como vimos, no se restringen al campo educativo; y tercero, ignora los múltiples trabajos de la comunidad académica internacional que proporcionan evidencias y análisis sobre la trascendencia del BM en el campo educativo, a nivel mundial.

De facto, para dar dos ejemplos, en la introducción del libro Global Managerial Education Reforms and Teachers (2010: 2), donde escriben autores de varias nacionalidades, se subraya que los principales promotores de estas políticas en contextos de bajos ingresos son las organizaciones internacionales como el Banco Mundial y más aún, que la estrategia llamada Aprendizaje para

\footnotetext{
${ }^{4}$ Patronato Emilio Azcárraga Jean, José Ignacio Ávalos Hernández, Alejandro Baillères Gual, Agustín, Coppel Luken, Antonio del Valle Perochena, José Antonio Fernández Carbajal, Carlos Fernández González, Claudio X. González Guajardo, Pablo González Guajardo, Carlos Hank González, Sissi Harp Calderoni, Fernando Landeros Verdugo, Laura Diez Barroso de Laviada, Alicia Lebrija Hirschfeld, Alejandro Ramírez Magaña, Ignacio Deschamps González, Alejandro Legorreta González, Marcos Martínez Gavica, Carlos Rahmane Sacal, Daniel Servitje Montull, Eduardo Tricio Haro [véase (Mal)Gasto: Estado de la Educación en México, 2013].
} 
Todos (2011) o el libro, Haciendo el Trabajo de las Escuelas, publicado por el mismo Banco Mundial, representa, en gran medida, las creencias fundamentales y las principales herramientas de la política global de estas reformas gerenciales.

En El Secuestro de la Inteligencia en la Sociedad del Conocimiento (2006), encontré algunos pocos textos donde los académicos expresaban su preocupación por la cultura de la auditoría que se instauró particularmente en las universidades Británicas durante el gobierno de Margaret Tacher (Richardson, 2000); a la inversa, para hacer el presente trabajo, resultaría una tarea titánica leer todos los materiales que, desde que comenzó a circular el documento Learning For All, es decir hace solamente tres años, ha producido la comunidad académica, que contienen estudios de caso de las más diversas regiones y países que demuestran, en suma, como escriben (Klees, Steven J. et al., 2012: xv) también en la introducción de otro libro colectivo:

El entusiasmo del Banco Mundial por sus propias declaraciones políticas y consejos prácticos no ha ido acompañado del progreso sostenido en la implementación de la educación como un derecho humano o en el logro de la calidad de la educación. Regularmente, sus recomendaciones son un problema, no una solución.

Así el debate suscitado por el documento del Banco Mundial Aprendizaje para todos. Estrategia 2020, se expresa ya en una gran cantidad de respuestas críticas que en la forma de artículos, libros y libros colectivos, páginas webs que ha producido la comunidad académica internacional desde 2011 a la fecha, algunos de los cuales expondré muy brevemente aquí.

Así, el Banco Mundial realiza actividades crediticias y actividades no crediticias y éstas últimas corresponden a estudios económicos y sectoriales; asistencia técnica, informes sobre el desarrollo mundial y servicios de investigación y evaluaciones de impacto de los proyectos sociales. De facto, el Banco Mundial se define a sí mismo como una fuente crucial de asistencia financiera y técnica para los países en desarrollo. Más específicamente, en relación a México al abrir la página del Banco mundial se lee "Actualmente el portafolio del Banco Mundial en México está compuesto por 18 proyectos activos y 22 productos relacionados con servicios de conocimiento"s.

Claramente, que el banco haga recomendaciones no significa que estas se impongan y/o se cumplan "a la de ya", el propio Banco en SABER $(2013: 7,9)$ señala que proporciona orientación basada en la evidencia de las investigaciones disponibles y un enfoque para evaluar si existen o no esas políticas en un sistema educativo determinado y que su foco de atención es el diseño de políticas, más que su implementación la cual puede variar, como suele suceder "sobre el terreno".

Más aún, Nordtveit (2013: 23) analiza la estructura y el vocabulario de la estrategia Learning For All, señalando que el lenguaje aquí parece tener un carácter persuasivo "en cuanto a la estrategia, la persuasión es intencional; el Banco Mundial por supuesto quisiera que se adhieran a sus

\footnotetext{
${ }^{5}$ Véase http://www.bancomundial.org/temas/resenas/educacion.htm
} 
puntos de vista". Creo que podríamos decir lo mismo respecto a SABER que muestra evidencias científicas, desde donde emergen sugerencias y recomendaciones.

En contraste, la Ley General del Servicio Profesional Docente (en la siguiente tabla Ley) que pasará por un proceso discursivo y práctico antes de su realización, conserva el tono de los ordenamientos legales que le corresponde y como he dicho, en ella puede observarse una estrecha coincidencia entre los 8 objetivos de política docente del BM presentes en el documento SABER de 2013 aquí citado y de donde destacaré los aspectos que considero más relevantes y novedosos:

\begin{tabular}{|c|c|}
\hline SABER & $\begin{array}{l}\text { bjetivo 1.- Expectativas claras para los profesores. Para el cumplimiento de este objetivo se } \\
\text { comienda 1.- Expectativas claras de lo que los estudiantes deberían conocer y ser capaces } \\
\text { hacer y, 2.- Orientación útil sobre el uso del tiempo de los docentes, agregando aquí que las } \\
\text { vestigaciones demuestran que en los sistemas educativos de alto rendimiento, los profesores } \\
\text { dican más tiempo a la colaboración entre colegas, que al contacto con sus estudiantes (p. } \\
\text { ). }\end{array}$ \\
\hline LEY & $\begin{array}{l}\text { Artículo 1. La presente Ley es reglamentaria de la fracción III del Artículo 3o. de la Constitución } \\
\text { Política de los Estados Unidos Mexicanos, rige el Servicio Profesional Docente y establece } \\
\text { los criterios, los términos y condiciones para el Ingreso, la Promoción, el Reconocimiento y } \\
\text { la Permanencia en el Servicio. } \\
\text { Art. 12. Las funciones docentes, de dirección de una Escuela o de supervisión de la Educación } \\
\text { Básica y Media Superior impartida por el Estado y sus Organismos Descentralizados deberán } \\
\text { orientarse a brindar educación de calidad y al cumplimiento de sus fines. Quienes desempeñen } \\
\text { dichas tareas deben reunir las cualidades personales y competencias profesionales para } \\
\text { que dentro de los distintos contextos sociales y culturales promuevan el máximo logro de } \\
\text { aprendizaje de los educandos, conforme a los perfiles, parámetros e indicadores que garanticen } \\
\text { la idoneidad de los conocimientos, aptitudes y capacidades que correspondan. } \\
\text { Art. 14. para alcanzar los propósitos del Servicio Profesional Docente deben desarrollarse } \\
\text { perfiles, parámetros e indicadores que permitan, al menos, lo siguiente: IV. Considerar la } \\
\text { observancia de los calendarios y el debido aprovechamiento del tiempo escolar. } \\
\text { Art. 16. II. Organizar en cada Escuela los espacios físicos y de tiempo para intercambiar } \\
\text { experiencias, compartir proyectos, problemas y soluciones con la comunidad de docentes y } \\
\text { el trabajo en conjunto entre las escuelas de cada zona escolar, que permita la disponibilidad } \\
\text { presupuestal; así como aportar los apoyos que sean necesarios para su debido cumplimiento. } \\
\text { Art. } 62 \text {. En cada Escuela deberá integrarse una comunidad de docentes que trabaje } \\
\text { armónicamente y cumpla con el perfil adecuado. }\end{array}$ \\
\hline SABER & $\begin{array}{l}\text { bjetivo 2. Atraer a los mejores en la enseñanza, refiere a la creación de requisitos de ingreso } \\
\text { de un proceso selectivo que permita atraer a los candidatos con talento. Es de agregar que } \\
\text { incluye políticas relacionadas con la ventaja comparativa de conocimiento de la materia } \\
\text { formación inicial pedagógica del profesorado, porque las pruebas al respecto son todavía } \\
\text { ntrovertidas (p. 28). }\end{array}$ \\
\hline
\end{tabular}




\begin{tabular}{|c|c|}
\hline LEY & $\begin{array}{l}\text { Art. 21. El Ingreso al Servicio en la Educación Básica y Media Superior que imparta el Estado } \\
\text { y sus Organismos Descentralizados, se llevará a cabo mediante concursos de oposición, } \\
\text { preferentemente anuales... } \\
\text { Art. 24. Los concursos de oposición para el ingreso podrán participar todas las personas que } \\
\text { cumplanconelperfilrelacionadoconelnivel,tipo,modalidadymateriaeducativacorrespondiente; } \\
\text { privilegiando, en la Educación Básica el perfil pedagógico docente de los candidatos; también } \\
\text { se considerarán perfiles correspondientes a las disciplinas especializadas de la enseñanza. } \\
\text { Vigésimo Primero. } \\
\text { El Art. } 24 \text { de la presente Ley entrará en vigor para la Educación Básica a los dos años siguientes } \\
\text { a su publicación en el Diario Oficial de la Federación, entre tanto, las convocatorias para } \\
\text { concursos de oposición para el Ingreso a la Educación Básica serán sólo para los egresados de } \\
\text { las Normales y sólo en el caso de que no se cubran las vacantes mediante dichos concursos, se } \\
\text { emitirán convocatorias públicas abiertas. }\end{array}$ \\
\hline SABER & $\begin{array}{l}\text { Objetivo 3.- Preparación de los maestros con entrenamiento útil y experiencia Los maestros } \\
\text { necesitan conocimiento en la materia y conocimientos pedagógicos, habilidades de gestión } \\
\text { del aula y una gran experiencia práctica en la enseñanza. Adicionalmente (p. 28) se menciona } \\
\text { que investigación en sistemas educativos de alto desempeño muestra que la mayoría requieren } \\
\text { que los profesores tengan una cantidad considerable de experiencia en el aula antes de } \\
\text { convertirse en maestros independientes, y algunos de estos sistemas proporcionan orientación } \\
\text { y apoyo durante el primer y hasta segundo año en el trabajo. También en la meta } 7 \text { se agrega } \\
\text { que las investigaciones han encontrado que el apoyo de mentores, la asignación de tutores, } \\
\text { supervisores o entrenadores a los profesores de bajo rendimiento coadyuva a la efectividad } \\
\text { de los maestros (p. 33). }\end{array}$ \\
\hline LEY & $\begin{array}{l}\text { Art. 22. En la Educación Básica y Media Superior el Ingreso a una plaza docente } \\
\text { dará lugar a un Nombramiento Definitivo de base después de seis meses de } \\
\text { servicios sin nota desfavorable en su expediente, en términos de esta Ley. } \\
\text { Con el objeto de fortalecer las capacidades, conocimientos y competencias del Personal } \\
\text { Docente de nuevo Ingreso, durante un periodo de dos años tendrá el acompañamiento de } \\
\text { un tutor designado por la Autoridad Educativa o el Organismo Descentralizado, según } \\
\text { corresponda. }\end{array}$ \\
\hline SABER & $\begin{array}{l}\text { Objetivo 4. El emparejar competencias docentes con las necesidades de los estudiantes que } \\
\text { garantiza que los profesores trabajen en las escuelas donde sus habilidades son más necesarias } \\
\text { e importante para lograr equidad y eficiencia. Puede utilizarse para alcanzar este objetivo: (1) } \\
\text { Proporcionar incentivos (monetarios o de otro tipo) para atraer a los profesores a las llamadas } \\
\text { hard-to-staff schools, es decir las situadas en zonas económicamente deprimidas y/o aisladas. }\end{array}$ \\
\hline LEY & $\begin{array}{l}\text { Art. 36. Las promociones a que se refiere este Capítulo deberán incluir los criterios siguientes: } \\
\text { VI. Generar incentivos para atraer al Personal Docente con buen desempeño en el ejercicio } \\
\text { de su función a las escuelas que atiendan a los estudiantes provenientes de los hogares más } \\
\text { pobres y de las zonas alejadas a los centros urbanos. }\end{array}$ \\
\hline SABER & $\begin{array}{l}\text { 5. Liderazgo con fuertes Directores, donde se considera que los directores capaces pueden } \\
\text { ayudar a atraer y retener a maestros competentes, se espera que los directores apoyen los } \\
\text { procesos de instrucción práctica, exigiéndoles explícitamente proporcionar apoyo en la } \\
\text { evaluación de desempeño docente; pero no se recomienda que tengan incidencia directa en } \\
\text { los procesos de contratación o despidos o directamente en los montos salariales. } \\
\text { Por otra parte, debe asegurarse de que los individuos correctos sean seleccionados para } \\
\text { los cargos de dirección ya que son la clave para el éxito del sistema educativo; esto puede } \\
\text { garantizarse haciendo que estos puestos sean atractivos y que las personas tienen las } \\
\text { competencias necesarias para el trabajo de director (p. } 30 \text { ). }\end{array}$ \\
\hline
\end{tabular}




\begin{tabular}{|c|c|}
\hline LEY & $\begin{array}{l}\text { Art. 15. La evaluación interna deberá ser una actividad permanente, de carácter formativo y } \\
\text { tendiente al mejoramiento de la práctica profesional de los docentes y al avance continuo de } \\
\text { la Escuela y de la zona escolar. } \\
\text { Dicha evaluación se llevará a cabo bajo la coordinación y liderazgo del director. Los docentes } \\
\text { tendrán la obligación de colaborar en esta actividad. } \\
\text { Art. } 62 \text {. Las Autoridades Educativas y los Organismos Descentralizados darán aviso a los } \\
\text { directores de las escuelas del perfil de los docentes que pueden ser susceptibles de adscripción. } \\
\text { Por su parte, los directores deberán verificar que esos docentes cumplan con el perfil para los } \\
\text { puestos que deban ser cubiertos. } \\
\text { Las Autoridades Educativas y los Organismos Descentralizados estarán obligados a revisar la } \\
\text { adscripción de los docentes cuando los directores señalen incompatibilidad del perfil con las } \\
\text { necesidades de la Escuela, y efectuar el reemplazo de manera inmediata de acreditarse dicha } \\
\text { incompatibilidad. } \\
\text { Art. } 27 \text {. En la Educación Básica la Promoción a una plaza con funciones de dirección dará } \\
\text { lugar a un Nombramiento, sujeto a un periodo de inducción con duración de dos años } \\
\text { ininterrumpidos, dentro del cual el personal de que se trate deberá cursar los programas de } \\
\text { desarrollo de liderazgo y gestión escolar determinados por la Autoridad Educativa Local... } \\
\text { Al término del periodo de inducción, la Autoridad Educativa Local evaluará el desempeño del } \\
\text { personal para determinar si cumple con las exigencias propias de la función directiva. Si el } \\
\text { personal cumple con dichas exigencias, se le otorgará Nombramiento Definitivo. } \\
\text { Cuando en la evaluación se identifique la insuficiencia en el nivel de desempeño de las } \\
\text { funciones de dirección, el personal volverá a su función docente en la Escuela en que hubiere } \\
\text { estado asignado. }\end{array}$ \\
\hline SABER & $\begin{array}{l}\text { Objetivo 6: Monitoreo de enseñanza y aprendizaje. Se menciona que existen evidencias que } \\
\text { la evaluación de estudiantes y maestros se vincula con la mejora de la calidad educativa y se } \\
\text { invita a considerar si los profesores están obligados a participar en dichas evaluaciones (p. } \\
\text { 32). } \\
\text { Previamente se señala que para documentar las políticas de evaluación existente es importante } \\
\text { abordar, entre otros aspectos, que los profesores deben ser evaluados por la escuela y por } \\
\text { autoridades externas (p. 20.) y que el impacto de cualquier evaluación está relacionado con } \\
\text { sus consecuencias legales. Se agrega que la evaluación es útil para premiar a los buenos } \\
\text { maestros y apoyar o castigar a los ineficaces. Las consecuencias pueden incluir, por ejemplo, } \\
\text { aumentar el salario de buenos maestros; reasignar los estudiantes con mayores dificultades de } \\
\text { aprendizaje a los buenos profesores; prestación adicional de apoyo profesional a maestros con } \\
\text { bajo rendimiento; o quitar a los maestros de bajo rendimiento del salón de clases. }\end{array}$ \\
\hline
\end{tabular}




\begin{tabular}{|c|c|}
\hline LEY & $\begin{array}{l}\text { Art. 52. Las Autoridades Educativas y los Organismos Descentralizados deberán evaluar } \\
\text { el desempeño docente y de quienes ejerzan funciones de dirección o de supervisión en la } \\
\text { Educación Básica y Media Superior que imparta el Estado. } \\
\text { La evaluación a que se refiere el párrafo anterior será obligatoria. El Instituto determinará } \\
\text { su periodicidad, considerando por lo menos una evaluación cada cuatro años y vigilará su } \\
\text { cumplimiento. } \\
\text { En la evaluación del desempeño se utilizarán los perfiles, parámetros e indicadores y los } \\
\text { instrumentos de evaluación que para fines de Permanencia sean definidos y autorizados } \\
\text { conforme a esta Ley. } \\
\text { Los Evaluadores que participen en la evaluación del desempeño deberán estar evaluados y } \\
\text { certificados por el Instituto. } \\
\text { Art. } 53 \text {. Cuando en la evaluación a que se refiere el Art. anterior se identifique la insuficiencia } \\
\text { en el nivel de desempeño de la función respectiva, el personal de que se trate se incorporará a } \\
\text { los programas de regularización que la Autoridad Educativa o el Organismo Descentralizado } \\
\text { determine, según sea el caso. Dichos programas incluirán el esquema de tutoría correspondiente. } \\
\text { El personal sujeto a los programas a que se refiere el párrafo anterior, tendrá la oportunidad } \\
\text { de sujetarse a una segunda oportunidad de evaluación en un plazo no mayor de doce meses } \\
\text { después de la evaluación a que se refiere el Art. } 52 \text {, la cual deberá efectuarse antes del inicio } \\
\text { del siguiente ciclo escolar o lectivo. } \\
\text { De ser insuficientes los resultados en la segunda evaluación, el evaluado se reincorporará a los } \\
\text { programas de regularización para sujetarse a una tercera evaluación que se llevará a cabo en } \\
\text { un plazo no mayor de doce meses. } \\
\text { En caso de que el personal no alcance un resultado suficiente en la tercera evaluación que } \\
\text { se le practique, se darán por terminados los efectos del Nombramiento correspondiente } \\
\text { sin responsabilidad para la Autoridad Educativa o el Organismo Descentralizado, según } \\
\text { corresponda. }\end{array}$ \\
\hline SABER & $\begin{array}{l}\text { Objetivo 7: Apoyo a los maestros para mejorar la instrucción. } \\
\text { Con el fin de mejorar constantemente la instrucción práctica, maestros y escuelas necesitan } \\
\text { ser capaces de analizar determinados desafíos que se enfrentan en el aula y tener acceso a } \\
\text { información sobre las mejores prácticas; para abordar estos desafíos es apropiado recibir apoyo } \\
\text { externo específico adaptado a sus necesidades y proveer desarrollo profesional adicional a los } \\
\text { maestros. }\end{array}$ \\
\hline LEY & $\begin{array}{l}\text { Art. 13. VII. Garantizar la formación, capacitación y actualización continúa del Personal } \\
\text { del Servicio Profesional Docente a través de políticas, programas y acciones específicas } \\
\text { y el articulo Décimo Primero. señala El programa de Carrera Magisterial continuará en } \\
\text { funcionamiento hasta en tanto entre en vigor el programa a que se refiere el Art. } 37 \text { de esta } \\
\text { Ley, cuya publicación deberá hacerse a más tardar el } 31 \text { de mayo del año } 2015 \text {. }\end{array}$ \\
\hline SABER & $\begin{array}{l}\text { Objetivo 8. Motivar el desempeño de los profesores, en este aspecto se subraya la importancia } \\
\text { de los incentivos para el buen desempeño y la creación de mecanismos de rendición de } \\
\text { cuentas, estableciendo ambos sanciones y premios y se invita a considerar si hay requisitos } \\
\text { tales como las evaluaciones de desempeño para permanecer en la enseñanza, y si los profesores } \\
\text { pueden ser despedidos con causa justa, como mala conducta, abuso infantil, ausentismo o bajo } \\
\text { rendimiento (p. 34). }\end{array}$ \\
\hline
\end{tabular}




\begin{tabular}{|c|c|}
\hline LEY & $\begin{array}{l}\text { Art. 13. El Servicio Profesional Docente tiene los propósitos siguientes: VIII. Desarrollar } \\
\text { un programa de estímulos e Incentivos que favorezca el desempeño eficiente del servicio } \\
\text { educativo y contribuya al reconocimiento escolar y social de la dignidad magisterial. } \\
\text { Art. } 76 \text {. Con el propósito de asegurar la continuidad en el servicio educativo, el servidor } \\
\text { público del sistema educativo nacional, el Personal Docente y el Personal con Funciones de } \\
\text { Dirección o de Supervisión en la Educación Básica y Media Superior que incumpla con la } \\
\text { asistencia a sus labores por más de tres días consecutivos o discontinuos, en un periodo de } \\
\text { treinta días naturales, sin causa justificada será separado del servicio sin responsabilidad para } \\
\text { la Autoridad Educativa o para el Organismo Descentralizado, y sin necesidad de que exista } \\
\text { resolución previa del Tribunal Federal de Conciliación y Arbitraje o sus equivalentes en las } \\
\text { entidades federativas, aplicando para ello el procedimiento previsto en el Art. } 75 \text { de esta Ley. }\end{array}$ \\
\hline
\end{tabular}

\section{Crítica de la comunidad académica}

En La Ley del Servicio Profesional Docente de 2013, puede observarse con claridad la impronta del Banco Mundial, así, la ley atañe al tipo de reformas que la comunidad académica en México y a nivel internacional atribuyen contenidos no directamente educativos ya que conserva un carácter laboral, gerencial, administrativo y empresarial que resulta comprensible en el marco de la dinámica económica explicada por Aboites (2007). Así, se pretende mejorar los niveles de aprendizaje, pero, en su análisis y recomendaciones, no se reflexiona sobre el proceso educativo. Promueven la medición estandarizada de los aspectos de la educación en función de la calidad, pero este concepto queda indefinido.

Podría decirse a lo sumo que en la estrategia del BM, la educación es un medio no un fin y que impulsa la visión del estudiante como mano de obra que debe ser apta al mercado laboral, al sector privado que, adicionalmente y como señala Nordtveit (2013), es concebido como el socio principal en la aplicación del "servicio" de la educación.

La ley aquí referida solamente rige el ámbito de la educación que imparte el Estado (Art. 3 ), pero al igual que otras reformas a nivel global, pretende transformar la manera en la que opera introduciendo las reglas, valores y técnicas del sector privado; ella implica una nueva cultura del desempeño que Bourdieu (1998) analiza como una nueva forma del ejercicio del poder; pero también una concepción de lo humano como apropiado a la lógica empresarial y comercial del servicio/cliente.

Dentro de esta mirada los incentivos que en la práctica individualizan el monto del salario, no se acompañan de mejoras en las condiciones laborales y al igual que las evaluaciones, cuyo efecto en la calidad educativa es ampliamente dudoso, incrementan la competencia entre profesores, escuelas, países.

Subyace en esta ley también la construcción imaginaria del ser humano como esencialmente egoísta, que actúa motivado por interés personal y funciona en razón a premios y castigos; de este modo, se erosiona la identidad de la profesión docente, pero también la subjetividad del maestro, como subraya Ball (2003), no solamente cambian lo que el maestro es, sino también lo que el maestro debería ser. 
Se puede sintetizar diciendo que esta ley contiene las 4 paradojas mencionadas por Verger et al. (2010:1-19), la primera, consiste en que subraya la importancia de los maestros en la calidad de la educación, pero al mismo tiempo los trata como objetos de intervención y socava su autonomía frente al estado y las familias de los estudiantes. La segunda paradoja se refiere al hecho de que implica más responsabilidades para los maestros, incluso cuando su preparación y condiciones de trabajo podrían ser peores pero, al mismo tiempo, promueve su desprofesionalización.

La tercera paradoja se relaciona con la utilización selectiva de las pruebas, así, por un lado, promueven reformas administrativas incluso cuando están conscientes de el hecho de que todavía no hay evidencia concluyente de su impacto positivo en los resultados del aprendizaje y por el otro, parecen ignorar las evidencias de países donde sus recomendaciones son muy marginales, no obstante los resultados son los mejores. Así, en países como Finlandia, Canadá o Cuba -con los mejores resultados de aprendizaje en sus respectivas regiones- el trabajo docente es altamente profesionalizado, no existe un sistema de incentivos, etcétera, quisiera agregar un ejemplo en América Latina, particularmente en Colombia el Decreto 1278 del 19 de junio de 2002, la evaluación no satisfactoria trae como consecuencia el retiro del servicio; no obstante, en los resultados PISA 2012, obtuvo puntajes menores que México.

A nivel conceptual Heyneman (2013: 59) subraya que el BM ha perdido prestigio en el ámbito profesional, en la medida en la que se ha vuelto auto referencial, el autor hace una contabilidad de los textos que cita y encuentra que el BM ha incrementado, desde 1995 hasta 2011, la referencia a textos producidos por el propio BM como autor, por autores que laboran dentro del mismo BM o en otras agencias internacionales que comparten su perspectiva. Critica más radical es la que realiza Steiner-Khamsi (2012: 10) desde el irónico titulo For All By All? planteando que el BM no ha disminuido su rol financiero pero sí ha incrementado su rol de asesor global de políticas, hasta convertirse en un "super think tank" entre las agencias internacionales de ayuda, un banco de conocimientos cuyas evaluaciones de impacto son a veces más costosas que la "intervención"; de modo tal que la misma institución identifica un paquete de reformas, presta el dinero para su implementación y también evalúa su efectividad; así en lugar de definir primero el problema y buscar soluciones, los gobiernos locales se alinean a los detectados exteriormente por la agencia que financia su solución y continúa funcionando de modo impositivo, similar a los préstamos condicionados de las impopulares reformas de ajuste estructural impuestas por el BM y el FMI a los países receptores de donaciones y préstamos.

Finalmente, el cambio de gestión del sistema educativo que no es necesariamente más eficiente, resulta costoso, requiere mayores procesos burocráticos, etcétera.

A pesar de las evidencias mixtas y de las críticas, éstas recomendaciones se convierten en programas y leyes, no solamente porque corresponden a compromisos internacionales, sino también porque tienen gran cobertura mediática, como los resultados de PISA; por otra parte, los datos y análisis del BM proporcionan prestigio a los funcionarios de los países en desarrollo así como una explicación fácil que atribuye a los profesores el fracaso educativo. 
La Trascendencia del Banco Mundial en La Ley General del Servicio Profesional Docente. Elementos para el Análisis

\section{Bibliografía}

Audit Cultures Anthropological Studies in Accountability, Ethics and the Academy (2000). Londres y Nueva York: Marilyn Strathern.

Aboites Aguilar, Hugo (2007). "Tratado de Libre Comercio y Educación Superior: El caso de México, un antecedente para América Latina”. En: Perfiles educativos, vol. 29 núm. 118, México.

Bourdieu, Pierre (1998). "Utopia of endless exploitation. The essence of Neoliberalism". En: Le Monde diplomatic. Diciembre.

Calderón, David (2012). “¿Protestar por ser evaluados?”. Extraído en octubre 2013 de: http://www. mexicanosprimero.org/maestros/blog-mexicanos-primero/362-iprotestar-por-ser-evaluados. html

Coraggio, J. L. (1997). Las propuestas del Banco Mundial para la educación: ¿sentido oculto o problemas de concepción? En: Coraggio, J. L. y R. M. Torres. La educación según el Banco Mundial. Buenos Aires: Miño y Dávila-CEM.

Global Partnership for Education - Status of Donor Contributions as of 30 April 2013. Extraído en marzo de 2014 de: www.globalpartnership.org/.../global-partnership-ed

The Case for Investment 2011-2014. Extraído en octubre de 2014 de: http://www.globalpartnership.org/content/case-investment-2011-2014

10 Key Results. Extraído en mayo de 2014 de: http:/www.globalpartnership.org/10-key-data-results

Grupo del Banco Mundial Resumen Ejecutivo (2011). Aprendizaje para todos; Invertir en los conocimientos y las capacidades de las personas para fomentar el desarrollo; Estrategia de Educación 2020. Extraído en marzo de 2013 de http:/www-wds;worldbank;org/external/default/ WDSContentServer/WDSP/IB/2011/09/15/000386194_20110915015838/Rendered/INDEX/ 644870WP00SPAN00Box0361538B0PUBLIC0;txt

Heyneman, Stephen P (2012). "When Models becomes Monopoly: The Making of Education Policy at The World Bank". En: Collins, Christopher y Alexander Wiseman (eds.). Education Strategy in the Developing World: Revising the World Bank's Education Policy. International perspective and society. Volumen 16.

Klees, Steven J. y Joel Samoff (eds.) (2013). "World Bank and Education: Ideological Premises and Ideological Conclusions". En: The World Bank and Education Critiques and Alternatives. Comparative International Education A Diversity of Voices. Volume 14. Rotterdam/Boston/ Taipei: Sense Publishers.

Mantilla, Lucia (2006). "El Secuestro de la Inteligencia en la Sociedad del Conocimiento”. En: Peredo Merlo, Alicia, Lucia Mantilla Gutiérrez y Patricia García Guevara (coords.). Tendencias de la educación Superior en México. México: Universidad de Guadalajara.

MÉXICO “EVALUACIÓN DE ‘EDUCACIÓN PARA TODOS 2000” elaborada por La Dirección General de Relaciones Internacionales de la Secretaría de Educación Pública. México, enero 
del 2000; Extraído en marzo de 2013, de http://www;unesco;org/education/wef/countryreports/mexico/contents;html.

Mexicanos Primero (2013). (Mal)Gasto: Estado de la Educación en México. Extraído en septiembre de 2013 de: http://www.mexicanosprimero.org/images/stories/malgasto/malgasto_estadode-la-educacion-en-mexico 2013.pdf

Nordtveit, Bjorn H. (2013). "World Bank Poetry: How the Education Strategy 2020 Imagines the World”. En: Klees, Steven J. y Joel Samoff (eds.). The World Bank and Education Critiques and Alternatives. Comparative International Education A Diversity of Voices. Volume 14. Rotterdam/Boston/Taipei: Sense Publishers.

OCDE (2010). Acuerdo de cooperación México-OCDE para mejorar la calidad de la educación de las escuelas mexicanas. Mejorar las escuelas: estrategias para la acción en México. Establecimiento de un marco para la evaluación e incentivos para docentes: Consideraciones para México. Extraído de: http://www.oecd.org/edu/school/46216786.pdf

Richardson, Peter D. (2000). "Audit Culture and Anthropology". Journal of the Royal Anthropological Institute. Vol. 6.

Roseli, Pauline, Steer Esbet, Katie Smith y Asma Zubairi (2013) "Financing For Global Education Opportunities For Multilateral Action. A Report Prepared For The UN Special Envoy for Global Education for the High-level Roundtable on Learning for All: Coordinating the Financing and Delivery of Education. Action Center for Universal Education at Brookings. Unesco. Education for All global monitoring report". Extraído en mayo de 2014 de: http://www.brookings.edu/research/reports/2013/09/financing-global-education

Skilbeck, Malcolm (2000). "Education for All Global Synthesis". International Consultative Forum on Education for All Unesco. Extraído en marzo 2013 de: http://unesdoc;unesco;org/ images/0012/001200/120058e;pdf

Steiner-Khamsi, Gita (2012). "For All by All? The World Bank's Global Framework for Education”. En: Klees, Steven J. y Joel Samoff (eds.) (2013). The World Bank and Education Critiques and Alternatives. Comparative International Education A Diversity of Voices. Volume 14. Rotterdam/Boston/Taipei: Sense Publishers.

Torres, Rosa María (2000). "Una década de Educación para Todos: La tarea pendiente"; publicado en español en 2000. Montevideo: FUM-TEP y en 2000 Caracas: Editorial Laboratorio Educativo. Publicado en inglés [One Decade of Education for All: The Challenge Ahead] en 2000. Buenos Aires: IIPE-UNESCO. Extraído en marzo de 2013 de:

http://www;cerlalc;org/redplanes/secciones/biblioteca/docs_marco_rosatorres;pdf

Torres, Rosa María. “¿Qué pasó en el Foro Mundial de la Educación?”. Extraído en marzo 2013 de: http://www;iizdvv;de/index;php?article_id=473\&clang=3

The World Bank (Updated June 14, 2012). Human Development Network. SABER Systems Approach for Better Education Results; What matters most in teacher policies? A framework for 
La Trascendencia del Banco Mundial en La Ley General del Servicio Profesional Docente. Elementos para el Análisis

building a more effective teaching profession. Extraído el 15 de marzo de 2013 de: https://ww w:google;com;mx/\#q=SABER+Systems+Approach+for+Better+Education+Results;++What + matters + most + in + teacher + policies $\% 3 \mathrm{~F}+\mathrm{A}+$ framework + for + building $+\mathrm{a}+$ more + effective + te aching + profession +

— SABER-Teachers-Country Reports. Extraído en mayo 13 de 2014 de:

http://saber.worldbank.org/index.cfm?indx $=9 \& p d=1$

UNESCO (1990). Declaración Mundial sobre Educación para Todos. Satisfacción de las Necesidades Básicas de Aprendizaje. Nueva York. Extraído en marzo de 2013 de: http:// unesdoc; unesco;org/images/0012/001275/127583s;pdf

Verger, Antoni, Hülya Kosar Altinyelken y Mireille De Koning (eds.) (2013). Global Managerial Education Reforms and Teachers. Education International Research Institute IS Academic Program; Education International. Bélgica.

World Bank Group (2011). Learning for All Education Strategy 2020; Investing in People's Knowledge and Skills to Promote Development. Extraído en octubre de 2013 de: http:// web;worldbank;org/WBSITE/EXTERNAL/TOPICS/EXTEDUCATION/0, contentMDK:22 474207 menuPK:282402 pagePK:210058 piPK:210062 theSitePK:282386,00;html

Recibido: 16/05/14

Dictaminado: 03/11/14

Corregido: 26/11/14

Aceptado: 30/11/14 